\title{
XVII. The law of partition of kinetic energy
}

\section{S.H. Burbury}

To cite this article: S.H. Burbury (1900) XVII. The law of partition of kinetic energy, Philosophical Magazine Series 5, 49:297, 226-228, DOI: 10.1080/14786440009463838

To link to this article: http://dx.doi.org/10.1080/14786440009463838

$$
\text { 册 Published online: } 21 \text { Apr } 2009 .
$$

Submit your article to this journal $x$

Q View related articles ¿ 
six times as well as the other. The curves have been drawn by an approximate method which depends on the fact that near each angular point $A, C$, the equation which tranforms the kite-shaped figure of fig. 3 into the square of fig. 2 takes the form $z^{\prime}=\mathrm{A} z^{n}$. The accuracy of the diagrams will therefore be least near the centre of each square $\triangle B C D$, where the position of an equipotential line may be in error by 10 per cent. of its distance from the next one.

Professor Lamb has pointed out to me that by superposing a second flux, the direction of which is at right angles to the first, on the first, a flux with stream and equipotential lines through the centres of the squares parallel to their sides is obtained. The conductivity of the medium for this flux will be readily seen to be given again by the formula

$$
k=\sqrt{k_{1} k_{2}} \text {. }
$$

The equipotential lines (or the stream-lines) for a strip of the medium, when one constituent conducts six times as well as the other, are shown in fig. 6 .

Fig. 6.

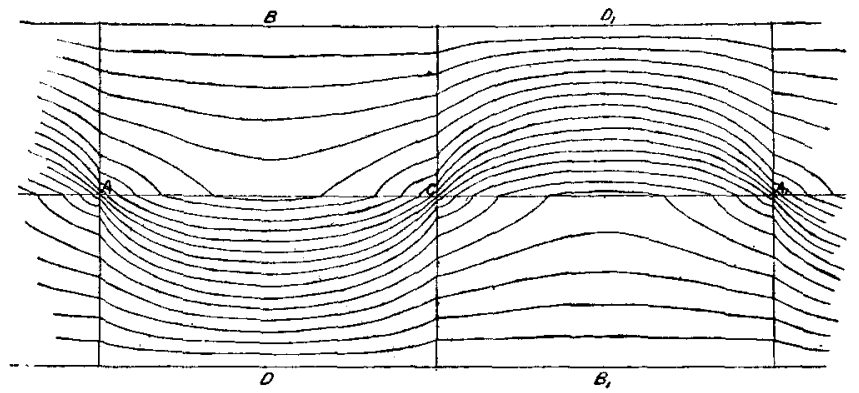

Since there are four directions in the medium in which the conductivity has the above value, the conductivity of the medium for a flux in any direction has the same value, $i$. $e$. equation (4) gives the conductivity of a compound medium in the general case where the constituents are present in the form of long prisms with their axes perpendicular to the planes of flow.

XVII. The Law of Partition of Kinetic Energy. By S. H. BurburY*.

I ORD RAYLEIGH'S article $†$ seems to raise the question what is the enunciation of the Maxwell-Boltzmann law of equal partition of energy. It cannot mean that in every case in which a number of particles of unequal masses are in stationary motion each particle has the same mean

* Communicated by the Author. $\dagger$ Supra, p. 98. 
kinetic energy. For it is easy to construct a system in which that is not true. For instance, suppose two parallel fixed elastic planes, $\mathrm{P}$ and $\mathrm{Q}$, and $\mathrm{PQ}$ a common normal. Let $m$ and $\mathrm{M}$ be two elastic spheres of unequal masses, each baving its centre in the line PQ. Initially let $m$ be touching the plane $\mathrm{P}$ and be projected in direction $\mathrm{PQ}$. Simultaneously let $\mathrm{M}$ be touching the plane $\mathrm{Q}$ and be projected in direction QP. The two spheres will collide, having their line of centres in $\mathrm{PQ}$, with the point of contact, say, at R. If the momenta are equal and opposite, the two spheres will, after collision, retrace their respective courses, will rebound normally from the two planes, and will again collide at the same place, and so on for ever. Since the momenta are equal and the masses unequal, the kinetic energies are unequal. Yet the motion is stationary. The condition of stationary motion is then not a sufficient foundation for the theorem of equal partition of energy. What further condition is necessary or sufficient?

It is generally understood that the theorem is proved in the Kinetic Theory of Gases, the simplest form of which may be stated thos:-

A very great number of elastic spheres are moving in a bounded space, and freely by their encounters or collisions exchanging energy with one another. Let no forces act except during collisions between two spheres, or between a sphere and the bounding surface, supposed perfectly elastic. Let $f(u, v, w) d u d v d w$ or $f . d u d v d w$ be at any instant the number of spheres $m$ whose component velocities lie within the limits

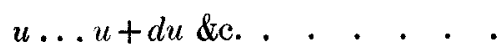

Similarly, let $\mathrm{F}(\mathrm{U}, \mathrm{V}, \mathrm{W}) d \mathrm{U} d \mathrm{~V} d \mathrm{~W}$ or $\mathrm{F} \cdot d \mathrm{U} d \mathrm{~V} d \mathrm{~W}$ be the number of spheres $M$ whose component velocities lie at the same instan $t$ within the limits

$$
\mathrm{U} \ldots \mathrm{U}+d \mathrm{U} \& \mathrm{dc} \text {. }
$$

If $m$ and $\mathrm{M}$, having these velocities, are properly situated with respect to one another, they will within the time $d t$ after the given instant collide with each other, and assume new velocities denoted by the accented letters $u^{\prime} \& c . U^{\prime} \& c$. where

$$
\begin{aligned}
m\left(u^{2}+v^{2}+w^{2}\right)+\mathrm{M}\left(\mathrm{U}^{4}+\mathrm{V}^{2}+\mathrm{W}^{2}\right) & =m\left(u^{12}+v^{12}+w^{12}\right) \\
& +\mathrm{M}\left(\mathrm{U}^{\prime 2}+\mathrm{V}^{\prime 2}+\mathrm{W}^{12}\right) .
\end{aligned}
$$

Boltzmann now says that it is necessary to make a special assumption. And the assumption he does make is that the motion is, and continues to be, molecular ungeordnet (see Vorlesungen über" Gas \& lieorie, Part I.). 'The state " molecular ungeordnet" is not completely defined, perhaps because if it 
were so defined, it would eo ipso facto cease to be " molecular ungeordnet." But the working assumption which he actually makes use of in the special case, as a deduction from hì general assumption, is the same as that used by Dr. Watson and other writers, namely that, regarding $f$ and $\mathrm{F}$ as the chances that a molecule shall have velocities between the limits (1) or (2), these chances are independent of the relative position of the molecules-at least that this is the case for molecules approaching collision. Hence $f$ and $\mathrm{F}$ being independent, the number per unit of volume and time of collisions between members of the class $f$ and members of the class $\mathrm{F}$ is proportional to $F f$. And from this, by a well-known method, it is proved that

$$
\begin{aligned}
& f^{\prime}=c \epsilon^{-h m\left(u^{2}+v^{2}+w^{2}\right)} \\
& \mathbf{F}=\mathrm{C} \epsilon^{-h \mathbf{M}\left(\mathrm{U}^{2}+\mathrm{V}^{2}+\mathrm{W}^{2}\right)} ;
\end{aligned}
$$

and therefore $m \overline{u^{2}}=\mathrm{M} \overline{\mathrm{U}^{2}} \& \mathrm{ce}$, which is the law.

But the question arises whether the state of things assumed by Boltzmann to exist, from which the theorem of equal partition of energy follows as a mathematical deduction, can exist, and continue to exist, in fact in a finite system of molecules left to their own mutual actions for infinite time undisturbed from without.

I think Lord Rayleigh's argument, p. 109 et seq., requires some assumptions :-

(1) Systems on the same path move independently of one another.

(2) Systems cannot exchange paths; or if they can we must assume a law of interchange.

I think there may be other constants besides E, e. $g$. if two elastic spheres with velocities $u_{1}, u_{2}$, \&c. collide, $u_{1} u_{2}+v_{1} v_{2}+w_{1} u_{2}$ is constant as well as $\mathrm{E}$.

Does not the method prove too much? namely that not merely the law of equal partition (p. 115), but in effect the distribution $\boldsymbol{e}^{-\boldsymbol{h} \mathbb{R}}$ must hold for all states of matter.

XVIII. Notes on the Electromagnetic Theory of Light.-I. Geometrical Properties of the Wave-Surface \&c. II. Reflexion and Refraction at the Boundary of Crystals, treated by a Theorem of Sir William howan Hamilton. By Prof. A. MoAulay, University of Tasmania *.

1. Geometrical Properties of the Wave-Surface \&s. M R. OLIVER HEAVISIDE (' Electrical Papers,' vol. ii. 1 p. 1 ; Phil. Mag. xix. June 1885, p. 397) first, J believe, investigated the properties of the general electro-

* Communicated by the Author. To be read at the Meeting of the Australasian Association for the Advancement of Science, January 1900. 